\title{
Properties of Classical and Quantum Jensen-Shannon Divergence
}

\author{
Jop Briët* and Peter Harremoës $\}^{\dagger}$ \\ Centrum Wiskunde \& Informatica, Science Park 123, 1098 XG Amsterdam, The Netherlands
}

(Dated: October 22, 2018)

\begin{abstract}
Jensen-Shannon divergence (JD) is a symmetrized and smoothed version of the most important divergence measure of information theory, Kullback divergence. As opposed to Kullback divergence it determines in a very direct way a metric; indeed, it is the square of a metric. We consider a family of divergence measures $\left(\mathrm{JD}_{\alpha}\right.$ for $\left.\alpha>0\right)$, the Jensen divergences of order $\alpha$, which generalize JD as $\mathrm{JD}_{1}=\mathrm{JD}$. Using a result of Schoenberg, we prove that $\mathrm{JD}_{\alpha}$ is the square of a metric for $\alpha \in(0,2]$, and that the resulting metric space of probability distributions can be isometrically embedded in a real Hilbert space. Quantum Jensen-Shannon divergence (QJD) is a symmetrized and smoothed version of quantum relative entropy and can be extended to a family of quantum Jensen divergences of order $\alpha\left(\mathrm{QJD}_{\alpha}\right)$. We strengthen results by Lamberti et al. by proving that for qubits and pure states, $\mathrm{QJD}_{\alpha}^{1 / 2}$ is a metric space which can be isometrically embedded in a real Hilbert space when $\alpha \in(0,2]$. In analogy with Burbea and Rao's generalization of JD, we also define general QJD by associating a Jensen-type quantity to any weighted family of states. Appropriate interpretations of quantities introduced are discussed and bounds are derived in terms of the total variation and trace distance.
\end{abstract}

PACS numbers: 89.70.Cf, 03.67.-a

\section{INTRODUCTION}

For two probability distributions $P=\left(p_{1}, \ldots, p_{n}\right)$ and $Q=\left(q_{1}, \ldots, q_{n}\right)$ on a finite alphabet of size $n \geq 2$, Jensen-Shannon divergence (JD) is a measure of divergence between $P$ and $Q$. It measures the deviation between the Shannon entropy of the mixture $(P+Q) / 2$ and the mixture of the entropies, and is given by

$$
\mathrm{JD}(P, Q)=H\left(\frac{P+Q}{2}\right)-\frac{1}{2}(H(P)+H(Q)) .
$$

Attractive features of this function are that it is everywhere defined, bounded, symmetric and only vanishes when $P=Q$. Endres and Schindelin 1 proved that it is the square of a metric, which we call the transmission metric $\left(\mathrm{d}_{\mathrm{T}}\right)$. This result implies, for example, that Banach's fixed point theorem holds for the space of probability distributions endowed with the metric $\mathrm{d}_{\mathrm{T}}$. A natural way to extend Jensen-Shannon divergence is to consider a mixture of $k$ probability distributions $P_{1}, \ldots, P_{k}$, with weights $\pi_{1}, \ldots, \pi_{k}$, respectively. With $\pi=\left(\pi_{1}, \ldots, \pi_{k}\right)$, we can then define the general Jensen divergence as

$$
\mathrm{JD}^{\pi}\left(P_{1}, \ldots, P_{k}\right)=H\left(\sum_{i=1}^{k} \pi_{i} P_{i}\right)-\sum_{i=1}^{k} \pi_{i} H\left(P_{i}\right) .
$$

This was already considered by Gallager [2] in 1968, who proved that, for fixed $\pi$, this is a convex function in $\left(P_{1}, \cdots, P_{k}\right)$. Further identities and inequalities were derived by Lin and Wong [3, 4, and Topsøe [5]. It has found

*Electronic address: jop.briet@cwi.nl.

$\dagger^{\dagger}$ Electronic address: P.Harremoes@cwi.nl. a variety of important applications: Sibson [6] showed that it has applications in biology and cluster analysis, Wong and You [7] used it as a measure of distance between random graphs, and recently, Rosso et al. used it to quantify the deterministic vs. the stochastic part of a time series [8]. For its statistical applications we refer to El-Yaniv et al. 9] and references therein.

Burbea and Rao 10 introduced another level of generalization, based on more general entropy functions. For an interval $I$ in $\mathbb{R}$ and a function $\phi: I \rightarrow \mathbb{R}$, they define the $\phi$-entropy of $x \in I^{n}$ (where $I^{n}$ denotes the Cartesian product of $n$ copies of $I$ ) as

$$
H_{\phi}(x)=-\sum_{i=1}^{n} \phi\left(x_{i}\right)
$$

Based on this, they define the generalized mutual information measure as

$$
\mathrm{JD}_{\phi}^{\pi}\left(P_{1}, \ldots, P_{k}\right)=H_{\phi}\left(\sum_{i=1}^{k} \pi_{i} P_{i}\right)-\sum_{i=1}^{k} \pi_{i} H_{\phi}\left(P_{i}\right),
$$

for which they established some strong convexity properties. If $k=2, I=[0,1]$ and $\phi$ is the function $x \rightarrow \frac{1}{\alpha-1}\left(x^{\alpha}-x\right)$, then $H_{\phi}$ defines the entropy of order $\alpha$. In this case, Burbea and Rao proved that $\mathrm{JD}_{\phi}^{\pi}$ is convex for all $\pi$, if and only if $\alpha \in[1,2]$, except if $n=2$ when convexity holds if and only if $\alpha \in[1,2]$ or $\alpha \in[3,11 / 3]$.

We focus on the functions $\mathrm{JD}_{\phi}^{\pi}$, where $k \geq 2, I=[0,1]$ and $\phi$ defines entropy of order $\alpha$. For ease of notation we write these as $\mathrm{JD}_{\alpha}^{\pi}$ if $k \geq 2$ and as $\mathrm{JD}_{\alpha}$ if $k=2$ and $\pi=(1 / 2,1 / 2)$.

Shannon entropy is additive in the sense that the entropy of independent random variables, defined as the entropy of their joint distribution, is the sum of their 
individual entropies. Like Shannon entropy Rényi of order $\alpha$ entropy is additive but in general Rényi entropy is not convex [26]. The power entropy of order $\alpha$ is a monotone function of Rényi entropy but, contrary to Rényi entropy it is a concave function which is what we are interested in. The study of power entropy dates back to J.H. Havrda and F. Charvat [27. Since then it was rediscovered independently several times [11, 12, 28, but we have chosen the more neutral term entropy of order $\alpha$ rather than calling it Hravda-Chervat-Lindhardt-Nielsen-AczélDar'oczy-Tsallis entropy. Entropy of order $\alpha$ is not additive (unless $\alpha=1$ ). This is one of the reasons why this function is used by physicists in attempts to model long range interaction in statistical mechanics, cf. Tsallis 11 and followers (can be traced from a bibliography maintained by Tsallis).

Martins et al. 13, 14, 15, 16, give non-extensive (i.e. non-additive) generalizations of JD based on entropies of order $\alpha$ and an extension of the concept of convexity to what they call $q$-convexity. For these functions they extend Burbea and Rao's results in terms of $q$-convexity.

Distance measures between quantum states, which generalize probability distributions, are of great interest to the field of quantum information theory [17, [18, 19, 20, 21]. They play a central role in state discrimination and in quantifying entanglement. For example, the quantum relative entropy of two states $\rho_{1}$ and $\rho_{2}$, given by $S\left(\rho_{1} \| \rho_{2}\right)=-\operatorname{Tr} \rho_{1}\left(\ln \rho_{1}-\ln \rho_{2}\right)$, is a commonly used distance measure. (For a review of its basic properties and applications see [22]). However, it is not symmetric and does not obey the triangle inequality. As an alternative, Lamberti et al. [21, 23, 24] proposed to use the (classical) JD as a distance function for quantum states, but also introduced a quantum version based on the von Neumann entropy, which we denote by QJD. Like its classical variant, it is everywhere defined, bounded, symmetric and zero only when the inputs are two identical quantum states. They prove that it is a metric on the set of pure quantum states and that it is close to the Wootter's distance and its generalization introduced by Braunstein and Caves [18. Whether the metric property holds in general is unknown.

As an analogue to $\mathrm{JD}_{\alpha}^{\pi}$ for quantum states, we introduce the general quantum Jensen divergence of order $\alpha$ $\left(\mathrm{QJD}_{\alpha}^{\pi}\right)$. In the limit $\alpha \rightarrow 1$ we obtain the "von Neumann version":

$$
\operatorname{QJD}^{\pi}\left(\rho_{1}, \ldots, \rho_{k}\right)=S\left(\sum_{i=1}^{k} \pi_{i} \rho_{i}\right)-\sum_{i=1}^{k} \pi_{i} S\left(\rho_{i}\right),
$$

where $S(\rho)=-\operatorname{Tr} \rho \ln \rho$ is the von Neumann entropy. For $k=2$ and $\pi=(1 / 2,1 / 2)$ one obtains the quantum Jensen divergence of order $\alpha\left(\mathrm{QJD}_{\alpha}\right)$, which generalizes QJD as $\lim _{\alpha \rightarrow 1}$ QJD $_{\alpha}=$ QJD.

\section{Our results.}

We extend the results of Endres and Schindelin, concerning the metric property of JD, and those of Lamberti et al., concerning the metric property of QJD, as follows:

- Denoting the set of probability distributions on a set $X$ by $M_{+}^{1}(X)$, we prove that for $\alpha \in(0,2]$, the pair $\left(M_{+}^{1}(X), \mathrm{JD}_{\alpha}^{1 / 2}\right)$ is a metric space which can be isometrically embedded in a real separable Hilbert space.

- Denoting the set of quantum states on qubits (2dimensional Hilbert spaces) by $\mathcal{B}_{+}^{1}\left(\mathcal{H}_{2}\right)$ and the set of pure-states on $d$-dimensional Hilbert spaces by $\mathcal{P}\left(\mathcal{H}_{d}\right)$, we prove that for $\alpha \in(0,2]$, the pairs $\left(\mathcal{B}_{+}^{1}\left(\mathcal{H}_{2}\right), \mathrm{QJD}_{\alpha}^{1 / 2}\right)$ and $\left(\mathcal{P}\left(\mathcal{H}_{d}\right), \mathrm{QJD}_{\alpha}^{1 / 2}\right)$ are metric spaces which can be isometrically embedded in a real separable Hilbert space.

- We show that these results do not extend to the cases $\alpha \in(2,3)$ and $\alpha \in\left(\frac{7}{2}, \infty\right)$. More precisely, we show that, for $\alpha \in(2,3)$, neither $\mathrm{JD}_{\alpha}$ nor $\mathrm{QJD}_{\alpha}$ can be the square of a metric, and for $\alpha \in\left(\frac{7}{2}, \infty\right)$, isometric embedding in a real Hilbert space is impossible (though the metric property may still hold).

\section{Techniques.}

To prove our positive results, we evoke a theorem by Schoenberg which links Hilbert space-embeddability of a metric space $(X, d)$ to the property of negative definiteness (defined in Section IV). We prove that for $\alpha \in(0,2]$, $\mathrm{JD}_{\alpha}$ satisfies this condition for every set of probability distributions, and that $\mathrm{QJD}_{\alpha}$ satisfies this condition for every set of qubits or pure-states.

\section{A. Interpretations of $\mathrm{JD}^{\pi}$ and $\mathrm{QJD}^{\pi}$}

\section{Channel capacity.}

A discrete memoryless channel is a system with input and output alphabets $X$ and $Y$ respectively, and conditional probabilities $p(y \mid x)$ for the probability that $y \in Y$ is received when $x \in X$ is sent. For a discrete memoryless channel with $|X|=k$, input distribution $\pi$ over $X$ and conditional distributions $P_{x}(y)=p(y \mid x)$, we have that $\mathrm{JD}^{\pi}\left(P_{x_{1}}, \ldots, P_{x_{k}}\right)$ in fact gives the transmission rate. (See for example [25].) Inspired by this fact, we call the metric defined by the square root of JD the transmission metric and denote it by $\mathrm{d}_{\mathrm{T}}$.

A quantum channel has classical input alphabet $X$, and an encoding of every element $x \in X$ into a quantum state $\rho_{x}$. A receiver decodes a message by performing a 
measurement with $|Y|$ possible outcomes, on the state he or she obtained. For a quantum channel with $|X|=k$, input distribution $\pi$ over $X$, and encoded elements $\rho_{x}$, Holevo's Theorem [29] says that the maximum transmission rate of classical information (the classical channel capacity) is at most $\operatorname{QJD}^{\pi}\left(\rho_{x_{1}}, \ldots, \rho_{x_{k}}\right)$. Holevo 30, and Schumacher and Westmoreland [31] proved that this bound is also asymptotically achievable.

\section{Data compression and side information.}

Let $X=[k]$ be an input alphabet and for each $i \in X$ let $P_{i}$ be a distribution over output alphabet $Y$ with $|Y|=n$. Consider a setting where a sender uses a weighting $\pi$ over $X$, and a receiver who has to compress the received output data losslessly. We call the receiver's knowledge of which distribution $P_{i}$ is used at any time the side information, and difference between the average number of nats (units based on the natural logarithm instead of bits) used for the encoding when the side information is known, and when it is not known, the redundancy. In [32, this setting is referred to as the switching model.

If the receiver always knows which input distribution is used, then for each distribution $P_{i}$, he or she can apply the optimal compression encoding given by $H\left(P_{i}\right)$. Hence, if the receiver has access to the side information, the average number of nats, that the optimal compression encoding uses is given by $\sum_{i=1}^{k} \pi_{i} H\left(P_{i}\right)$.

However, if the receiver does not know when which input distribution is used, he or she always has to use the same encoding. We say that a compression encoding $C$ corresponds to an input distribution $Q$, if $C$ is optimal for $Q$ (i.e., the number of nats used is $H(Q)$ ). If the sender transmits an infinite sequence of letters $y_{1} y_{2} \ldots$ , picked according to distribution $P_{i}$, and the receiver compresses it using an encoding $C$ which corresponds to distribution $Q$, then the average number of used nats is given by $\sum_{j=1}^{n} P_{i}\left(y_{j}\right) \ln \frac{1}{Q\left(y_{j}\right)}$.

Hence, with the weighting $\pi_{1}, \ldots, \pi_{k}$, we get the redundancy

$$
\begin{aligned}
R(Q):= & \sum_{i=1}^{k}\left(\pi_{i} H\left(P_{i}\right)-\sum_{j=1}^{n} \pi_{i} P_{i}\left(y_{j}\right) \ln \frac{1}{Q\left(y_{j}\right)}\right) \\
& =\sum_{i=1}^{k} \pi_{i} D\left(P_{i} \| Q\right),
\end{aligned}
$$

a weighted average of Kullback divergences between the $P_{i}$ 's and $Q$. The compensation identity states that for $\bar{P}=\sum_{i=1}^{k} \pi_{i} P_{i}$, the equality

$$
\sum_{i=1}^{k} \pi_{i} D\left(P_{i} \| Q\right)=\sum_{i=1}^{k} \pi_{i} D\left(P_{i} \| \bar{P}\right)+D(\bar{P} \| Q)
$$

holds for any distribution $Q$, cf. [33, 34].
It follows immediately that $Q=\bar{P}$ is the unique argmin-distribution for $R(Q)$, and that $\mathrm{JD}^{\pi}\left(P_{1}, \ldots, P_{k}\right)$ is the corresponding minimum value.

Analogously in a quantum setting, let $X=[k]$ be an input alphabet, and for each $i \in X$ let $\rho_{i}$ be a state on an output Hilbert space $\mathcal{H}_{Y}$. We can think of a sender who uses the weighting $\pi$ of distributions $X$, but a receiver who has to compress the states on $\mathcal{H}_{Y}$ using as few qubits as possible.

Schumacher [35] showed that the mean number of qubits necessary to encode a state $\rho_{i}$ is given by $S\left(\rho_{i}\right)$. Later, Schumacher and Westmoreland [36] introduced a quantum encoding scheme, in which an encoding $C_{Q}$ that is optimal (i.e., requires the least number of qubits) for a state $\sigma$ requires on average $S\left(\rho_{i}\right)+S\left(\rho_{i} \| \sigma\right)$ qubits to encode $\rho_{i}$. Hence, when the receiver uses $C_{Q}$ as the encoding, the mean redundancy is $R(\sigma):=\sum_{i=1}^{k} \pi_{i} S\left(\rho_{i} \| \sigma\right)$. Let $\bar{\rho}=\sum_{i=1}^{k} \pi_{i} \rho_{i}$. The quantum analogue of 2 is given by Donald's identity [37]:

$$
\sum_{i=1}^{k} \pi_{i} S\left(\rho_{i} \| \sigma\right)=\sum_{i=1}^{k} \pi_{i} S\left(\rho_{i} \| \bar{\rho}\right)+S(\bar{\rho} \| \sigma),
$$

from which it follows that $\sigma=\bar{\rho}$ is the argmin-state that the receiver should code for, and that $\operatorname{QJD}^{\pi}\left(\rho_{1}, \ldots, \rho_{k}\right)$ is the minimum redundancy.

\section{PRELIMINARIES AND NOTATION}

In this section we fix notation to be used throughout the paper. We also provide a concise overview of those concepts from quantum theory which we need. For an extensive introduction we refer to [38].

\section{A. Classical information theoretic quantities}

We write $[n]$ for the set $\{1,2, \ldots, n\}$. The set of probability distributions supported by $\mathbb{N}$ is denoted by $M_{+}^{1}(\mathbb{N})$ and the set supported by $[n]$ is denoted by $M_{+}^{1}(n)$. We associate with probability distributions $P, Q \in M_{+}^{1}(n)$ point probabilities $\left(p_{1}, \ldots, p_{n}\right)$ and $\left(q_{1}, \ldots, q_{n}\right)$, respectively. Entropy of order $\alpha \neq 1$, Shannon entropy and Kullback divergence are given by

$$
\begin{aligned}
S_{\alpha}(P) & :=\frac{1-\sum_{i=1}^{n} p_{i}^{\alpha}}{\alpha-1}, \\
H(P) & :=-\sum_{i=1}^{n} p_{i} \ln p_{i}
\end{aligned}
$$

and

$$
D(P \| Q):=\sum_{i=1}^{n} p_{i} \ln \frac{p_{i}}{q_{i}}
$$


respectively. Note that $\lim _{\alpha \rightarrow 1+} S_{\alpha}(P)=H(P)$. For two-point probability distributions $P=(p, 1-p)$ we let $s_{\alpha}(p)$ denote $S_{\alpha}(p, 1-p)$.

\section{B. Quantum theory}

1. States.

The $d$-dimensional complex Hilbert space, denoted by $\mathcal{H}_{d}$, is the space composed of all $d$-dimensional complex vectors, endowed with the standard inner product. A physical system is mathematically represented by a Hilbert space. Our knowledge about a physical system is expressed by its state, which in turn is represented by a density matrix (a trace-1 positive matrix) acting on the Hilbert space. The set of density matrices on a Hilbert space $\mathcal{H}$ is denoted by $\mathcal{B}_{+}^{1}(\mathcal{H})$ [51. Rank-1 density matrices are called pure-states. Systems described by two-dimensional Hilbert spaces are called qubits. As the eigenvalues of a density matrix are always positive real numbers that sum to one, a state can be interpreted as a probability distribution over pure-states. Hence, sets of states with a complete set of common eigenvectors can be interpreted as probability distributions on the same set of pure-states. States thus generalize probability distributions. This interpretation is not possible when a common basis does not exist. Two states $\rho$ and $\sigma$ have a set of common eigenvectors if and only if they commute; i.e. $\rho \sigma=\sigma \rho$.

\section{Measurements.}

Information about a physical system can be obtained by performing a measurement on its state. The most general measurement with $k$ outcomes is described by $k$ positive matrices $A_{1}, \ldots, A_{k}$, which satisfy $\sum_{i=1}^{k} A_{i}=I$. This is a special case of the more general concept of a positive operator valued measure (POVM, see for example [38]). The probability that a measurement $A$ of a system in state $\rho$ yields the $i$ 'th outcome is $\operatorname{Tr}\left(A_{i} \rho\right)$. Hence, the measurement yields a random variable $A(\rho)$ with $\operatorname{Pr}\left[A(\rho)=\lambda_{i}\right]=\operatorname{Tr}\left(A_{i} \rho\right)$. Naturally, the measurement operators and quantum states should act on the same Hilbert space.

\section{Quantum information theoretic quantities}

For states $\rho, \sigma \in \mathcal{B}_{+}^{1}(\mathcal{H})$, we use the quantum version of entropy of order $\alpha$, von Neumann entropy and quantum relative entropy, given by

$$
S_{\alpha}(\rho):=\frac{1-\operatorname{Tr}\left(\rho^{\alpha}\right)}{\alpha-1}
$$

$$
S(\rho):=-\operatorname{Tr}(\rho \ln \rho)
$$

and

$$
S(\rho \| \sigma):=\operatorname{Tr} \rho \ln \rho-\operatorname{Tr} \rho \ln \sigma,
$$

respectively. Note that $\lim _{\alpha \rightarrow 1^{+}} S_{\alpha}(\rho)=S(\rho)$. We refer to 39] for a discussionof quantum relative entropy.

\section{DIVERGENCE MEASURES}

\section{A. The general Jensen divergence}

Let us consider a mixture of $k$ probability distributions $P_{1}, \ldots, P_{k}$ with weights $\pi_{1}, \ldots, \pi_{k}$ and let $\bar{P}=$ $\sum_{i=1}^{k} \pi_{i} P_{i}$. Jensen's inequality and concavity of Shannon entropy implies that

$$
H\left(\sum_{i=1}^{k} \pi_{i} P_{i}\right) \geq \sum_{i=1}^{k} \pi_{i} H\left(P_{i}\right)
$$

When entropies are finite, we can subtract the right-hand side from the left-hand side and use this as a measure of how much Shannon entropy deviates from being affine. This difference is called the general Jensen-Shannon $d i$ vergence and we denote it by $\operatorname{JD}^{\pi}\left(P_{1}, \ldots, P_{k}\right)$, where $\pi=\left(\pi_{1}, \ldots, \pi_{k}\right)$. One finds that

$$
H\left(\sum_{i-1}^{k} \pi_{i} P_{i}\right)-\sum_{i=1}^{k} \pi_{i} H\left(P_{i}\right)=\sum_{i=1}^{k} \pi_{i} D\left(P_{i} \| \bar{P}\right)
$$

and therefore

$$
\mathrm{JD}^{\pi}\left(P_{1}, \ldots, P_{k}\right)=\sum_{i=1}^{k} \pi_{i} D\left(P_{i} \| \bar{P}\right)
$$

In the general case when entropies may be infinite the last expression can be used, but we will focus on the situation where the distributions are over a finite set and in this case we can use the left-hand side of (7).

Jensen divergence of order $\alpha$ is defined by the formula

$$
\mathrm{JD}_{\alpha}^{\pi}\left(P_{1}, \ldots, P_{k}\right)=S_{\alpha}\left(\sum_{i=1}^{k} \pi_{i} P_{i}\right)-\sum_{i=1}^{k} \pi_{i} S_{\alpha}\left(P_{i}\right) .
$$

Similarly, if $\rho_{1}, \ldots, \rho_{k}$ are states on a Hilbert space we define

$$
\mathrm{QJD}^{\pi}\left(\rho_{1}, \ldots, \rho_{k}\right)=\sum_{i=1}^{k} \pi_{i} S\left(\rho_{i} \| \bar{\rho}\right)
$$

where $\bar{\rho}=\sum_{i=1}^{k} \pi_{i} \rho_{i}$. For states on a finite dimensional 
Hilbert space we have

$$
\operatorname{QJD}^{\pi}\left(\rho_{1}, \ldots, \rho_{k}\right)=S\left(\sum_{i=1}^{k} \pi_{i} \rho_{i}\right)-\sum_{i=1}^{k} \pi_{i} S\left(\rho_{i}\right) .
$$

The quantum Jensen divergence of order $\alpha$ is defined by

$$
\operatorname{QJD}_{\alpha}^{\pi}\left(\rho_{1}, \ldots, \rho_{k}\right)=S_{\alpha}\left(\sum_{i=1}^{k} \pi_{i} \rho_{i}\right)-\sum_{i=1}^{k} \pi_{i} S_{\alpha}\left(\rho_{i}\right) .
$$

\section{B. The Jensen divergence}

For even mixtures of two distributions, we introduce the notation $\mathrm{JD}_{\alpha}(P, Q)$ for $\mathrm{JD}_{\alpha}\left(\frac{1}{2} P+\frac{1}{2} Q\right)$. That is,

$$
\mathrm{JD}_{\alpha}(P, Q):=S_{\alpha}\left(\frac{P+Q}{2}\right)-\frac{1}{2} S_{\alpha}(P)-\frac{1}{2} S_{\alpha}(Q)
$$

For even mixtures of two states the QJD was defined in [23], to which we refer for some of its basic properties. We consider the order $\alpha$ version of this and write $\mathrm{QJD}_{\alpha}(\rho, \sigma)$ for $\mathrm{QJD}_{\alpha}\left(\frac{1}{2} \rho+\frac{1}{2} \sigma\right)$. That is,

$$
\operatorname{QJD}_{\alpha}(\rho, \sigma):=S_{\alpha}\left(\frac{\rho+\sigma}{2}\right)-\frac{1}{2} S_{\alpha}(\rho)-\frac{1}{2} S_{\alpha}(\sigma) .
$$

We refer to 10 and 111 simply as Jensen divergence of order $\alpha\left(\mathrm{JD}_{\alpha}\right)$ and quantum Jensen divergence of order $\alpha\left(\mathrm{QJD}_{\alpha}\right)$ respectively.

\section{METRIC PROPERTIES}

In this section we borrow most of the notational conventions and definitions from Deza and Laurent [40. We refer to this book, to Berg, Christensen and Ressel [48, and to Blumenthal [41] for extensive introductions to the used results. Like Berg, Christensen and Ressel [48] we shall use the expressions "positive and negative definite" for what most textbook would call "positive and negative semi-definite".

Definition 1. For a set $X$, a function $d: X \times X \rightarrow \mathbb{R}$ is called a distance if for every $x, y \in X$ :

1. $d(x, y) \geq 0$ with equality if $x=y$.

2. $d$ is symmetric: $d(x, y)=d(y, x)$.

The pair $(X, d)$ is then called a distance space. If in addition to 1 and 2 , for every triple $x, y, z \in X$, the function d satisfies

3. $d(x, y)+d(x, z) \geq d(y, z)$ (the triangle inequality), then $d$ is called a pseudometric and $(X, d)$ a pseudometric space. If also, $d(x, y)=0$ holds if and only if $x=y$, then we speak of a metric and a metric space.
Our techniques to prove our embeddability results for $\mathrm{JD}_{\alpha}$ and $\mathrm{QJD}_{\alpha}$ are somewhat indirect. To provide some intuition, we briefly mention the following facts. Only Definition 11. Proposition [1] and Theorem 3 are needed for our proofs.

Work of Cayley and Menger gives a characterization of $\ell_{2}$ embeddability of a distance space in terms of CayleyMenger determinants. Given a finite distance space $(X, d)$, the Cayley-Menger matrix $\operatorname{CM}(X, d)$ is given in terms of the matrix $D_{i j}=d\left(x_{i}, x_{j}\right)$, for $x_{i}, x_{j} \in X$, and the all-ones vector $e$ :

$$
\operatorname{CM}(X, d):=\left(\begin{array}{cc}
D & e \\
e^{T} & 0
\end{array}\right)
$$

Menger proved the following relation between $\ell_{2}$ embeddability and the determinant of $\operatorname{CM}(X, d)$.

Proposition 1 (42]). Let $(X, d)$ be a finite distance space. Then $\left(X, d^{1 / 2}\right)$ is $\ell_{2}$ embeddable if and only if for every $Y \subseteq X$, we have $(-1)^{|Y|} \operatorname{det} \mathrm{CM}(Y, d) \geq 0$.

As an example, consider a distance space with $|X|=$ 3. If we set $a:=d\left(x_{1}, x_{2}\right)^{1 / 2}, b:=d\left(x_{1}, x_{3}\right)^{1 / 2}$ and $d\left(x_{2}, x_{3}\right)^{1 / 2}$, then we obtain

$$
\begin{aligned}
& -\operatorname{det} \operatorname{CM}(X, d)= \\
& (a+b+c)(a-b-c)(-a+b-c)(-a-b+c) .
\end{aligned}
$$

On the one hand, this at least zero if $d$ is a pseudometric, and hence pseudometric spaces on three points are $\ell_{2}$ embeddable. On the other hand, up to a factor $1 / 16$, the right-hand-side of 12 is the square of Heron's formula for the area of a triangle with edge-lengths $a, b$ and $c$. In general, Cayley-Menger determinants give the formulas needed to calculate the squared hypervolumes of higher dimensional simplices. Menger's result can thus be interpreted as saying that a distance space $\left(X, d^{1 / 2}\right)$ is $\ell_{2}$ embeddable if and only if every subset is a simplex with real hypervolume.

Returning to our example with $|X|=3$, we also have the following implication.

Proposition 2. Let $\left(\left\{x_{1}, x_{1}, x_{3}\right\}, d\right)$ be a distance space. Assume that for every $c_{1}, c_{2}, c_{3} \in \mathbb{R}$ such that $c_{1}+c_{2}+$ $c_{3}=0$, the distance function $d$ satisfies

$$
\sum_{i, j} c_{i} c_{j} d\left(x_{i}, x_{j}\right) \leq 0
$$

where the summation is over all pairs $i, j \in\{1,2,3\}$. Then $\left(\left\{x_{1}, x_{1}, x_{3}\right\}, d^{1 / 2}\right)$ is $\ell_{2}$ embeddable.

Proof: Let $a:=d\left(x_{1}, x_{2}\right)^{1 / 2}, b:=d\left(x_{1}, x_{3}\right)^{1 / 2}$ and $c:=$ $d\left(x_{2}, x_{3}\right)^{1 / 2}$. We first show that $(13)$ implies that $(12)$ is nonnegative. To this end, set $c_{1}=1, c_{2}=t, c_{3}=-t-1$ where $t$ is a real parameter. Then, if 13 holds, we get the inequality

$$
a^{2} t+b^{2} t(-t-1)+c^{2}(-t-1) \leq 0 .
$$


The nonnegativity of 12 follows from the fact that this inequality holds if and only if the discriminant of this second order polynomial is at least zero. The result now follows from Proposition 1 .

The basis of our positive results in this section is that, due to Schoenberg 43, 44, a more general version of Proposition 2 also holds. To state it concisely, we first define negative definiteness.

Definition 2 (Negative definiteness). Let $(X, d)$ be a distance space. Then $d$ is said to be negative defninite if and only if for all finite sets $\left(c_{i}\right)_{i \leq n}$ of real numbers such that $\sum_{i=1}^{n} c_{i}=0$, and all corresponding finite sets $\left(x_{i}\right)_{i \leq n}$ of points in $X$, it holds that

$$
\sum_{i, j} c_{i} c_{j} d\left(x_{i}, x_{j}\right) \leq 0
$$

In this case, $(X, d)$ is said to be a distance space of negative type.

The following theorem follows as a corollary of Schoenberg's theorem.

Theorem 3. Let $(X, d)$ be a distance space. Then $\left(X, d^{1 / 2}\right)$ can be isometrically embedded in a real separable Hilbert space if and only if $(X, d)$ is of negative type.

Note that if isometric embedding in a Hilbert space is possible, then the space must be a metric space. We define positive definiteness as follows.

Definition 3 (Positive definiteness). Let $X$ be a set and $f: X \times X \rightarrow \mathbb{R}$ a mapping. Then $f$ is said to be positive definite if and only if for all finite sets $\left(c_{i}\right)_{i \leq n}$ of real numbers and all corresponding finite sets $\left(x_{i}\right)_{i \leq n}$ of points in $X$, it holds that

$$
\sum_{i, j} c_{i} c_{j} f\left(x_{i}, x_{j}\right) \geq 0
$$

Because we are concerned with functions defined on convex sets, the following definition shall be useful.

Definition 4 (Exponential convexity). Let $X$ be a convex set and $\phi: X \rightarrow \mathbb{R}$ a mapping. Then $\phi$ is said to be exponentially convex if the function $X \times X \rightarrow \mathbb{R}$ given by $(x, y) \rightarrow \phi\left(\frac{x+y}{2}\right)$ is positive definite.

Normally exponential convexity is defined as positive definiteness of $\phi(x+y)$ (as is done in for instance [45]), but the definition given here allows the function $\phi$ only to be defined on a convex set.

\section{A. Metric properties of $\mathrm{JD}_{\alpha}$}

With Theorem 3 we prove the following for Jensen divergence of order $\alpha$.
Theorem 4. For $\alpha \in(0,2]$, the space $\left(M_{+}^{1}(\mathbb{N}), \mathrm{JD}_{\alpha}^{1 / 2}\right)$ can be isometrically embedded in a real separable Hilbert space.

Note that Theorem 4 implies that the same holds for $\mathrm{QJD}_{\alpha}$ for sets of commuting quantum states.

We use the following lemma to prove that $\mathrm{JD}_{\alpha}$ is negative definite for $\alpha \in(0,2]$. Theorem 4 then follows from this and Theorem 3 .

Lemma 1. For $\alpha \in(0,1)$, we have

$$
x^{\alpha}=\frac{1}{\Gamma(-\alpha)} \int_{0}^{\infty} \frac{e^{-x t}-1}{t^{\alpha+1}} d t
$$

where $\Gamma(\alpha)=\int_{0}^{\infty} t^{\alpha-1} e^{-t} d t$ is the Gamma function. For $\alpha \in(1,2)$, we have

$$
x^{\alpha}=\frac{1}{\Gamma(-\alpha)} \int_{0}^{\infty} \frac{e^{-x t}-(1-x t)}{t^{\alpha+1}} d t .
$$

Proof: Let $\gamma \in(-1,0)$. From the definition of the Gamma function, we have the following equality:

$$
z^{\gamma}=z^{\gamma} \frac{1}{\Gamma(-\gamma)} \int_{0}^{\infty} r^{-(\gamma+1)} e^{-r} d r
$$

By substituting $r=t z$ we get

$$
z^{\gamma}=\frac{1}{\Gamma(-\gamma)} \int_{0}^{\infty} \frac{e^{-z t}}{t^{\gamma+1}} d t
$$

Let $\beta \in(0,1)$ such that $\beta=\gamma+1$. Integrating $z^{\gamma}$ for $z$ from zero to $y$ and multiplying by $\gamma+1$ gives,

$$
\begin{aligned}
y^{\beta} & =(\gamma+1) \int_{0}^{y} z^{\gamma} d z \\
& =\frac{1}{\Gamma(-\beta)} \int_{0}^{\infty} \frac{e^{-y t}-1}{t^{\beta+1}} d t .
\end{aligned}
$$

Now let $\alpha \in(1,2)$ such that $\alpha=\beta+1$. Integrating $y^{\beta}$ and multiplying by $\beta+1$ gives the result.

$$
\begin{aligned}
x^{\alpha} & =(\beta+1) \int_{0}^{x} y^{\beta} d y \\
& =\frac{1}{\Gamma(-\alpha)} \int_{0}^{\infty} \frac{e^{-x t}-(1-x t)}{t^{\alpha+1}} d t .
\end{aligned}
$$

Lemma 2. For $\alpha \in(0,2]$, the distance space $\left(M_{+}^{1}, \mathrm{JD}_{\alpha}\right)$ is of negative type.

Proof: Let $\left(c_{i}\right)_{i \leq n}$ be a set of real numbers such that $\sum_{i=1}^{n} c_{i}=0$. For two probability distributions $P$ and $Q$, we have

$$
\mathrm{JD}_{\alpha}(P, Q)=S_{\alpha}\left(\frac{P+Q}{2}\right)-\frac{1}{2} S_{\alpha}(P)-\frac{1}{2} S_{\alpha}(Q) .
$$


Observe that for any real valued, single-variable function $f$, we have $\sum_{i, j} c_{i} c_{j} f\left(x_{i}\right)=0$. Hence, we only need to prove that the function

$$
S_{\alpha}\left(\frac{P+Q}{2}\right)=\frac{1}{\alpha-1}-\frac{1}{(\alpha-1)} \sum_{i}\left(\frac{p_{i}+q_{i}}{2}\right)^{\alpha}
$$

is negative definite for all $\alpha \in(0,2]$. From this decomposition of $S_{\alpha}$ into a sum over point probabilities it follows that we need to show that $x \curvearrowright x^{\alpha}$ is exponentially convex. Lemma 1 shows that for fixed $0<\alpha<1$ and fixed $1<\alpha<2$, the mapping $x \curvearrowright-x^{\alpha}$ can be obtained as the limit of linear combinations with positive coefficients of functions of the type $x \curvearrowright 1-e^{-t x}$ and $x \curvearrowright 1-e^{-t x}-t x$ respectively. Each such function is exponentially convex since the linear terms are, and for non-negative real numbers $x_{1}, \ldots, x_{n}$,

$$
\sum_{i, j} c_{i} c_{j}\left(-e^{-t\left(x_{i}+x_{j}\right)}\right)=-\left(\sum_{i=1}^{n} c_{i} e^{-t x_{i}}\right)^{2} \leq 0 .
$$

The case $\alpha=1$ follows by continuity. The case $\alpha=2$ also follows by continuity, but a direct proof without Lemma 1 is straightforward.

Proof of Theorem 4; Follows directly from Lemma 2 and Theorem 3 .

A constructive proof of Theorem 4 for $\mathrm{JD}_{1}$ (JD) is given by Fuglede [46, 47], who uses an embedding into a subset of a real Hilbert space defined by a logarithmic spiral.

\section{B. Metric properties of $\mathrm{QJD}_{\alpha}$ for qubits}

Using the same approach as above, we prove the following for quantum Jensen divergence of order $\alpha$ and states on two-dimensional Hilbert spaces.

Theorem 5. For $\alpha \in(0,2]$, the space

$$
\left(\mathcal{B}_{+}^{1}\left(\mathcal{H}_{2}\right), \mathrm{QJD}_{\alpha}^{1 / 2}\right)
$$

can be isometrically embedded in a real separable Hilbert space.

This is established by the following lemmas and Theorem 3 .

Lemma 3. Let $(V,\langle\cdot \mid \cdot\rangle)$ be a real Hilbert space with norm $\|\cdot\|_{2}=\langle\cdot \mid \cdot\rangle^{1 / 2}$. Then, $\left(V,\|\cdot\|_{2}^{2}\right)$ is a distance space of negative type.

Proof: The result follows immidiately if we expand the distance function $\|\cdot\|_{2}^{2}$ in terms of the inner product:

$$
\begin{gathered}
\sum_{i, j} c_{i} c_{j}\left\langle x_{i}-x_{j}, x_{i}-x_{j}\right\rangle \\
=\sum_{i, j} c_{i} c_{j}\left(\left\|x_{i}\right\|_{2}^{2}+\left\|x_{j}\right\|_{2}^{2}-2\left\langle x_{i}, x_{j}\right\rangle\right) \\
=2 \sum_{i} c_{i} \sum_{j} c_{j}\left\|x_{j}\right\|_{2}^{2}-2 \sum_{i, j} c_{i} c_{j}\left\langle x_{i}, x_{j}\right\rangle \\
=0-2 \sum_{i, j} c_{i} c_{j}\left\langle x_{i}, x_{j}\right\rangle \\
=-2\left\|\sum_{i} c_{i} x_{i}\right\|_{2}^{2} \leq 0
\end{gathered}
$$

Lemma 4. The distance space $\left(\mathcal{B}_{+}^{1}\left(\mathcal{H}_{2}\right)\right.$, QJD $\left.{ }_{\alpha}\right), \alpha \in$ $(0,2]$ is of negative type.

Proof: Using the same techniques as in the proof of Theorem 4, and the fact that Lemma 1 also holds when $x$ is a matrix, what has to be shown is that for $\rho \in \mathcal{B}_{+}^{1}\left(\mathcal{H}_{2}\right)$, the function $\rho \curvearrowright \operatorname{Tr}(\exp (-t \rho))$ is exponentially convex. Since $\rho$ acts on a two-dimensional Hilbert space, it has only two eigenvalues, $\lambda_{+}$and $\lambda_{-}$, that satisfy $\lambda_{+}+\lambda_{-}=1$ and $\lambda_{+}^{2}+\lambda_{-}^{2}=\operatorname{Tr}\left(\rho^{2}\right)$. A straightforward calculation gives

$$
\lambda_{+/-}=\frac{1}{2} \pm \frac{\left(2 \operatorname{Tr}\left(\rho^{2}\right)-1\right)^{1 / 2}}{2} .
$$

Plugging this into $\operatorname{Tr}(\exp (-t \rho))$ gives

$$
\begin{aligned}
\operatorname{Tr}\left(e^{-t \rho}\right) & =2 e^{-t / 2} \cosh \left(\frac{t}{2}\left(2 \operatorname{Tr}\left(\rho^{2}\right)-1\right)^{1 / 2}\right) \\
& =2 e^{-t / 2} \sum_{k=0}^{\infty} \frac{t^{2 k}}{(2 k) ! 4^{k}}\left(2 \operatorname{Tr}\left(\rho^{2}\right)-1\right)^{k}
\end{aligned}
$$

where the second equality follows form the Taylor expansion of hyperbolic cosine. The task can thus be reduced to proving that $\left(2 \operatorname{Tr}\left(\rho^{2}\right)-1\right)^{k}$ is exponentially convex for all $k \geq 0$. For this we can use the following theorem:

Theorem 6 ([48, Slight reformulation of Theorem 1.12]). Let $\phi_{1}, \phi_{2}: X \curvearrowright \mathbb{C}$ be exponentially convex functions. Then $\phi_{1} \cdot \phi_{2}$ is exponentially convex too.

This implies that proving it for $k=1$ suffices. The trace distance of two density matrices is defined as the Hilbert-Schmidt norm $\|\cdot\|_{2}$ of their difference. Since the Hilbert-Schmidt norm is a Hilbert-space metric, Lemma 3 implies that $\left(\rho_{1}, \rho_{2}\right) \curvearrowright\left\|\rho_{1}-\rho_{2}\right\|_{1}^{2}$ is negative definite and the equality

$\left\|\rho_{1}-\rho_{2}\right\|_{2}^{2}=\operatorname{Tr}\left(\rho_{1}-\rho_{2}\right)^{2}=2\left(\operatorname{Tr} \rho_{1}^{2}+\operatorname{Tr} \rho_{2}^{2}\right)-\operatorname{Tr}\left(\left(\rho_{1}+\rho_{2}\right)^{2}\right)$

implies that the function $\operatorname{Tr}\left(\left(\rho_{1}+\rho_{2}\right)^{2}\right)$ is positive definite. From this it follows that the function $2 \operatorname{Tr}\left(\rho^{2}\right)-1$ 
is exponentially convex.

Proof of Theorem 5; Follows directly from Lemma 4 and Theorem 3 .

\section{Metric properties of $\mathrm{QJD}_{\alpha}$ for pure-states}

Here we prove that $\mathrm{QJD}_{\alpha}$ is the square of a metric when restricted to pairs of pure-states. For a Hilbert space of dimension $d$ we denote the set of pure-states as $P\left(\mathcal{H}_{d}\right)$

Theorem 7. For $\alpha \in(0,2]$, the space $\left(P\left(\mathcal{H}_{d}\right)\right.$, QJD $\left.{ }_{\alpha}^{1 / 2}\right)$ can be isometrically embedded in a real separable Hilbert space.

Lemma 5. The distance space $\left(P\left(\mathcal{H}_{d}\right)\right.$, QJD $\left.{ }_{\alpha}\right), \alpha \in$ $(0,2]$ is of negative type.

Proof: Using the same techniques as in Theorem 4 , we have to prove that for $\rho \in P\left(\mathcal{H}_{d}\right)$, the function $\rho \curvearrowright \operatorname{Tr}(\exp (-t \rho))$ is exponentially convex. For $\rho_{1}, \rho_{2} \in$ $P\left(\mathcal{H}_{d}\right)$ such that $\rho_{1} \neq \rho_{2}$, the matrix $\frac{\rho_{1}+\rho_{2}}{2}$ has two nonzero eigenvalues, $\lambda_{+}$and $\lambda_{-}$, which can be calculated in the same way as above. In this case 16 reduces to

$$
\lambda_{ \pm}=\frac{1}{2} \pm \frac{1}{2}\left(\operatorname{Tr}\left(\rho_{1} \cdot \rho_{2}\right)\right)^{1 / 2} .
$$

When we plug this into $\operatorname{Tr}\left(\exp \left(-t\left(\rho_{1}+\rho_{2}\right)\right)\right)$, we get

$$
\begin{aligned}
\operatorname{Tr}\left(e^{\left.-2 t\left(\frac{\rho_{1}+\rho_{2}}{2}\right)\right)=}\right. & (n-2) \\
& +2 e^{-t} \cosh \left(t\left(\operatorname{Tr}\left(\rho_{1} \cdot \rho_{2}\right)\right)^{1 / 2}\right) \\
= & (n-2) \\
& +2 e^{-t} \sum_{k=0}^{\infty} \frac{t^{2 k}\left(\operatorname{Tr}\left(\rho_{1} \cdot \rho_{2}\right)\right)^{k}}{(2 k) !}
\end{aligned}
$$

where the $(n-2)$ term comes from the fact that $n-2$ of the eigenvalues are zero. We need to prove that $\left(\rho_{1}, \rho_{2}\right) \curvearrowright\left(\operatorname{Tr}\left(\rho_{1} \cdot \rho_{2}\right)\right)^{k}$ is positive definite for all integers $k \geq 0$. But Theorem 6 implies that we only need to prove it for $k=1$. Appealing to the trace distance, we have

$$
\left\|\rho_{1}-\rho_{2}\right\|_{1}^{2}=\operatorname{Tr} \rho_{1}^{2}+\operatorname{Tr} \rho_{2}^{2}-2 \operatorname{Tr}\left(\rho_{1} \cdot \rho_{2}\right),
$$

Since, by Lemma 3 this is negative definite, the result follows.

Proof of Theorem 7; Follows directly from Lemma 5 and Theorem 3 .

\section{Counter examples}

1. Metric space counter example for $\alpha \in(2,3)$.

To see that $\mathrm{JD}_{\alpha}$, and hence $\mathrm{QJD}_{\alpha}$, is not the square of a metric for all $\alpha$ we check the triangle inequality for the three probability vectors $P=(0,1), Q=(1 / 2,1 / 2)$ and $R=(1,0)$. We have

$$
\begin{aligned}
\mathrm{JD}_{\alpha}(P, Q) & =\mathrm{JD}_{\alpha}(Q, R) \\
& =S_{\alpha}(1 / 4,3 / 4)-\frac{S_{\alpha}(1 / 2,1 / 2)}{2}
\end{aligned}
$$

and

$$
\mathrm{JD}_{\alpha}(P, R)=S_{\alpha}(1 / 2,1 / 2) .
$$

The triangle inequality is equivalent to the inequality

$$
\begin{aligned}
0 & \geq-2 \mathrm{JD}_{\alpha}(P, Q)-2 \mathrm{JD}_{\alpha}(Q, R)+\mathrm{JD}_{\alpha}(P, R) \\
& =-4\left(S_{\alpha}(1 / 4,3 / 4)-\frac{S_{\alpha}(1 / 2,1 / 2)}{2}\right)+S_{\alpha}(1 / 2,1 / 2) \\
& =3 S_{\alpha}(1 / 2,1 / 2)-4 S_{\alpha}(1 / 4,3 / 4) \\
& =3 \frac{1-2(1 / 2)^{\alpha}}{\alpha-1}-4 \frac{1-(1 / 4)^{\alpha}-(3 / 4)^{\alpha}}{\alpha-1} \\
& =\frac{4(1 / 4)^{\alpha}+4(3 / 4)^{\alpha}-6(1 / 2)^{\alpha}-1}{\alpha-1} .
\end{aligned}
$$

We make the substitution $x=(1 / 2)^{\alpha}$ and assume $\alpha>1$ so the inequality is equivalent to

$$
4 x^{2}+4 x^{\frac{\ln 4-\ln 3}{\ln 2}}-6 x-1 \leq 0 .
$$

Define the function

$$
f(x)=4 x^{2}+4 x^{2-\frac{\ln 3}{\ln 2}}-6 x-1 .
$$

Then its first and second derivatives are given by

$$
\begin{aligned}
& f^{\prime}(x)=8 x+4\left(2-\frac{\ln 3}{\ln 2}\right) x^{1-\frac{\ln 3}{\ln 2}}-6 \\
& f^{\prime \prime}(x)=8+4\left(2-\frac{\ln 3}{\ln 2}\right)\left(1-\frac{\ln 3}{\ln 2}\right) x^{-\frac{\ln 3}{\ln 2}}
\end{aligned}
$$

and we see that $f^{\prime \prime}(x)=0$ has exactly one solution. Therefore $f$ has exactly one infliction point and the equation $f(x)=0$ has at most three solutions. Therefore the equation

$$
4(1 / 4)^{\alpha}+4(3 / 4)^{\alpha}-6(1 / 2)^{\alpha}-1=0
$$

has at most three solutions. It is straightforward to check that $\alpha=1, \alpha=2$ and $\alpha=3$ are solutions, so these are the only ones. Therefore the sign of

$$
\frac{4(1 / 4)^{\alpha}+4(3 / 4)^{\alpha}-6(1 / 2)^{\alpha}-1}{\alpha-1}
$$


is constant in the interval $(2,3)$ and plugging in any number will show that it is negative in this interval. Hence $\mathrm{JD}_{\alpha}$ cannot be a square of a metric for $\alpha \in(2,3)$.

\section{Counter examples for Hilbert space embeddability for}

$$
\alpha \in\left(\frac{7}{2}, \infty\right) \text {. }
$$

In the previous paragraph we showed that $\mathrm{JD}_{\alpha}$ and $\mathrm{QJD}_{\alpha}$ are not the squares of metric functions for $\alpha \in$ $(2,3)$. Hence, for $\alpha$ in this interval, Hilbert space embeddings are not possible. Here we prove a weaker result for $\alpha \in\left(\frac{7}{2}, \infty\right)$, using the Cayley-Menger determinant.

Theorem 8. The space

$$
\left(\mathcal{B}_{+}^{1}\left(\mathcal{H}_{d}\right),\left(\mathrm{JD}_{\alpha}\right)^{\frac{1}{2}}\right)
$$

is not Hilbert space embeddable for $\alpha$ in the interval $\left(\frac{7}{2}, \infty\right)$.

Note that this does not exclude the possibility that $\mathrm{JD}_{\alpha}$ is the square of a metric and that the same result holds for $\mathrm{QJD}_{\alpha}$,

Proof: Consider the four distributions

$$
\begin{aligned}
& \left(\frac{1}{2}-3 \varepsilon, \frac{1}{2}+3 \varepsilon\right), \\
& \left(\frac{1}{2}-\varepsilon, \frac{1}{2}+\varepsilon\right), \\
& \left(\frac{1}{2}+\varepsilon, \frac{1}{2}-\varepsilon\right), \\
& \left(\frac{1}{2}+3 \varepsilon, \frac{1}{2}-3 \varepsilon\right) .
\end{aligned}
$$

Then the Cayley-Menger determinant is

$$
\left|\begin{array}{ccccc}
s_{\alpha}\left(\frac{1}{2}-3 \varepsilon\right) & s_{\alpha}\left(\frac{1}{2}-2 \varepsilon\right) & s_{\alpha}\left(\frac{1}{2}-\varepsilon\right) & s_{\alpha}\left(\frac{1}{2}\right) & 1 \\
s_{\alpha}\left(\frac{1}{2}-2 \varepsilon\right) & s_{\alpha}\left(\frac{1}{2}-\varepsilon\right) & s_{\alpha}\left(\frac{1}{2}\right) & s_{\alpha}\left(\frac{1}{2}+\varepsilon\right) & 1 \\
s_{\alpha}\left(\frac{1}{2}-\varepsilon\right) & s_{\alpha}\left(\frac{1}{2}\right) & s_{\alpha}\left(\frac{1}{2}+\varepsilon\right) & s_{\alpha, 2}\left(\frac{1}{2}+2 \varepsilon\right) & 1 \\
s_{\alpha}\left(\frac{1}{2}\right) & s_{\alpha}\left(\frac{1}{2}+\varepsilon\right) & s_{\alpha}\left(\frac{1}{2}+2 \varepsilon\right) & s_{\alpha}\left(\frac{1}{2}+3 \varepsilon\right) & 1 \\
1 & 1 & 1 & 1 & 0
\end{array}\right|
$$

and if the four points are Hilbert space embeddable then this determinant is non-negative. The function $\varepsilon \rightarrow s_{\alpha}\left(\frac{1}{2}+\varepsilon\right)$ has a Taylor expansion given by

$$
\begin{aligned}
s_{\alpha}\left(\frac{1}{2}+\varepsilon\right)= & s_{\alpha}\left(\frac{1}{2}\right)+\frac{s_{\alpha}^{\prime \prime}\left(\frac{1}{2}\right)}{2} \varepsilon^{2}+ \\
& \frac{s_{\alpha}^{(4)}\left(\frac{1}{2}\right)}{24} \varepsilon^{4}+\frac{s_{\alpha}^{(6)}\left(\frac{1}{2}\right)}{720} \varepsilon^{4}+\varepsilon^{8} f(\varepsilon),
\end{aligned}
$$

where $f$ is some continous function of $\varepsilon$. This can be used to get the expansion of the Cayley-Menger determinant:

$$
\begin{aligned}
\mathrm{CM}=\frac{1}{8} s_{\alpha}^{(4)}\left(\frac{1}{2}\right) & \left(\left(s_{\alpha}^{(4)}\left(\frac{1}{2}\right)\right)^{2}-\right. \\
& \left.s_{\alpha}^{\prime \prime}\left(\frac{1}{2}\right) h_{\alpha}^{(6)}\left(\frac{1}{2}\right)\right) \varepsilon^{12}+\varepsilon^{14} g(\varepsilon)
\end{aligned}
$$

for some continuous function $g[52$. We have the following formula for the even derivatives of $s_{\alpha}$ :

$$
s_{\alpha}^{(2 n)}(x)=-\alpha^{\frac{2 n}{}}\left(x^{\alpha-2 n}+(1-x)^{\alpha-2 n}\right)
$$

and

$$
s_{\alpha}^{(2 n)}\left(\frac{1}{2}\right)=-\alpha^{\frac{2 n}{2}} 2^{2 n+1-\alpha} .
$$

If the Cayley-Menger determinant is positive for all small $\varepsilon$ then

$$
\left(s_{\alpha}^{(4)}\left(\frac{1}{2}\right)\right)^{2}-s_{\alpha}^{\prime \prime}\left(\frac{1}{2}\right) s_{\alpha}^{(6)}\left(\frac{1}{2}\right) \leq 0
$$

or equivalently

$$
\left(-\alpha^{-} 2^{5-\alpha}\right)^{2}-\left(-\alpha^{\underline{2}} 2^{3-\alpha}\right)\left(-\alpha^{-} 2^{7-\alpha}\right) \leq 0
$$

and

$$
\begin{aligned}
0 & \geq\left(\alpha^{\underline{4}}\right)^{2}-\left(\alpha^{\underline{2}}\right)\left(\alpha^{\underline{6}}\right) \\
& =\alpha^{\underline{2}} \alpha^{\underline{4}}((\alpha-2)(\alpha-3)-(\alpha-4)(\alpha-5)) \\
& =4 \alpha^{\underline{2}}(\alpha-2)(\alpha-3)\left(\alpha-\frac{7}{2}\right)
\end{aligned}
$$

Hence, the Cayley-Menger determinant is non-negative only for the intervals $[0,2]$ and $\left[3, \frac{7}{2}\right]$.

\section{RELATION TO TOTAL VARIATION AND TRACE DISTANCE}

The results of Section [V] indicate that interesting geometric properties are associated with $\mathrm{JD}_{\alpha}$ and $\mathrm{QJD}_{\alpha}$ when $\alpha \in(0,2]$.

\section{A. Bounds on $\mathrm{JD}_{\alpha}$}

For $\alpha \in(0,2]$. we bound $\mathrm{JD}_{\alpha}$ as follows:

Theorem 9. Let $P$ and $Q$ be probability distributions in $M_{+}^{1}(n)$, and let

$$
v:=\frac{1}{2} \sum_{i}\left|p_{i}-q_{i}\right| \in[0,2]
$$

denote their total variation. Then for $\alpha \in(0,2]$, we have $L \leq \mathrm{JD}_{\alpha}(P, Q) \leq U$, where: 
- For every $n \geq 2, L$ is given by

$$
L(P, Q)=s_{\alpha}\left(\frac{1}{2}\right)-s_{\alpha}\left(\frac{1}{2}+\frac{v}{4}\right) .
$$

- For every $n \geq 3, U$ is given by

$$
U_{n}(P, Q)=\frac{1}{\alpha-1}\left(\frac{1}{2}-\frac{1}{2^{\alpha}}\right)\|P-Q\|_{\alpha}^{\alpha} .
$$

- For $n=2, U$ is given by the tighter quantity

$$
U_{2}(P, Q)=s_{\alpha}\left(\frac{v}{4}\right)-\frac{1}{2} S_{\alpha, 2}\left(\frac{v}{2}\right) .
$$

Proof: We start with the lower bound. Let $\sigma$ denote a permutation of the elements in $[n]$ and let $\sigma(P)$ denote the probability vector where the point probabilities have been permuted according to $\sigma$. Clearly, the function $\mathrm{JD}_{\alpha}$ is invariant under such permutations of its arguments:

$$
\mathrm{JD}_{\alpha}(\sigma(P), \sigma(Q))=\mathrm{JD}_{\alpha}(P, Q) \text {. }
$$

Let $B$ denote the set of permutations $\sigma$ that satisfy

$$
p_{i} \geq q_{i} \Leftrightarrow p_{\sigma(i)} \geq q_{\sigma(i)}
$$

for all $i \in[n]$. Then, by the joint convexity of $\mathrm{JD}_{\alpha}$ for $\alpha \in[1,2]$ (as proved in [10]), we have

$$
\begin{aligned}
\mathrm{JD}_{\alpha}(P, Q) & =\frac{1}{|B|} \sum_{\sigma \in B} \mathrm{JD}_{\alpha}(\sigma(P), \sigma(Q)) \\
& \geq \mathrm{JD}_{\alpha}\left(\frac{1}{|B|} \sum_{\sigma \in B} \sigma(P), \frac{1}{|B|} \sum_{\sigma \in B} \sigma(Q)\right) .
\end{aligned}
$$

The distributions $\frac{1}{|B|} \sum_{\sigma \in B} \sigma(P)$ and $\frac{1}{|B|} \sum_{\sigma \in B} \sigma(Q)$ have the property that they are constant on two complementary sets, namely $\left\{i \in[n] \mid p_{i} \geq q_{i}\right\}$ and $\{i \in[n] \mid$ $\left.p_{i}<q_{i}\right\}$. Therefore, we may without loss of generality assume that $P$ and $Q$ are distributions on a two-element set. On a two-element set $P$ and $Q$ can be parametrized by $P=(p, 1-p)$ and $Q=(q, 1-q)$. If $\sigma_{2}$ denotes the transposition of the two elements then

$$
v=V\left(\frac{P+\sigma_{2}(Q)}{2}, \frac{Q+\sigma_{2}(P)}{2}\right)=2|p-q| .
$$

By 21 and 22 we get

$$
\begin{array}{r}
\mathrm{JD}_{\alpha}(P, Q) \geq \mathrm{JD}_{\alpha}\left(\frac{P+\sigma_{2}(Q)}{2}, \frac{Q+\sigma_{2}(P)}{2}\right) \\
=\mathrm{JD}_{\alpha}\left(\left(\frac{1}{2}+\frac{v}{4}, \frac{1}{2}-\frac{v}{4}\right),\left(\frac{1}{2}-\frac{v}{4}, \frac{1}{2}+\frac{v}{4}\right)\right) \\
=s_{\alpha}(1 / 2)-s_{\alpha}\left(\frac{1}{2}+\frac{v}{4}\right),
\end{array}
$$

and this lower bound is attained for two distributions on a two element set. Next we derive the general upper bound. Define distribution $\widetilde{P}$ on $[n] \times[3]$ such that for every $i \in[n]$,

$$
\begin{aligned}
& \widetilde{P}(i, 1)=\min \left\{p_{i}, q_{i}\right\} \\
& \widetilde{P}(i, 2)= \begin{cases}p_{i}-q_{i} & \text { if } p_{i}>q_{i} \\
0 & \text { otherwise }\end{cases} \\
& \widetilde{P}(i, 3)=0,
\end{aligned}
$$

and similarly define $\widetilde{Q}$ on $[n] \times[3]$ by

$$
\begin{aligned}
& \widetilde{Q}(i, 1)=\min \left\{p_{i} . q_{i}\right\} \\
& \widetilde{Q}(i, 2)=0, \\
& \widetilde{Q}(i, 3)= \begin{cases}q_{i}-p_{i} & \text { if } q_{i}>p_{i} \\
0 & \text { otherwise. }\end{cases}
\end{aligned}
$$

With these definitions we have $V(\widetilde{P}, \widetilde{Q})=V(P, Q)$. Using the data processing inequality and the definitions of $\widetilde{P}$ and $\widetilde{Q}$ it is straighforward to verify that

$$
\begin{aligned}
\operatorname{JD}_{\alpha}(P, Q) & \leq \operatorname{JD}_{\alpha}(\widetilde{P}, \widetilde{Q}) \\
& =\frac{1}{\alpha-1}\left(\frac{1}{2}-\frac{1}{2^{\alpha}}\right) \sum_{i=1}^{n}\left|p_{i}-q_{i}\right|^{\alpha} .
\end{aligned}
$$

This upper bound is attained on a three element set so we have

$$
U_{n}(P, Q)=\frac{1}{\alpha-1}\left(\frac{1}{2}-\frac{1}{2^{\alpha}}\right)\|P-Q\|_{\alpha}^{\alpha} .
$$

To get a tight upper bound on a two-element set a special analysis is needed. The cases $p>q$ and $p<q$ are treated separately, but the two cases work the same way. We will therefore assume that $p>q$. On a twoelement set parametrize $P$ and $Q$ by $P=(p, 1-p)$ and $Q=(q, 1-q)$. In this case we have the linear constraint $p-q=v / 2$. For a fixed value of $v$, we have that $\mathrm{JD}_{\alpha}$ is a convex function of $q$. Therefore the maximum is attained by an extreme point, i.e. a distribution where either $p$ or $q$ is either 0 or 1 . Without loss of generality we may assume that $q=0$ and that $p=v / 2$. This gives

$$
U_{2}(P, Q)=s_{\alpha}\left(\frac{v}{4}\right)-\frac{s_{\alpha}\left(\frac{v}{2}\right)}{2} .
$$

It is now straightforward to determine the exact form of the joint range of $V$ and $\mathrm{JD}_{\alpha}$.

Corollary 10. The joint range of $V$ and $\mathrm{JD}_{\alpha}$, denoted by $\Delta_{n}$, is a compact region in the plane bounded by a (Jordan) curve composed of two curves: The first curve is given by (18) with $V$ running from 2 to 0 . For $n=2$ the second curve is given by 19 with $v$ running from 0 to 2, and for $n=3$ the second curve is given by (20) with $v$ running from 0 to 2. 
Proof: Assume first that $n \geq 3$. By Theorem 9 we know that $\Delta_{n}$ is contained in the compact domain described. A continuous deformation of the lower curve into the upper bounding curve (i.e. a homotopy from the lower bounding curve to the upper bounding curve ) is given by $P_{t}$, $Q_{t}$ for $t \in[0,1]$, where

$$
\begin{aligned}
\left(\begin{array}{c}
P_{t} \\
Q_{t}
\end{array}\right)(v)= & (1-t)\left(\begin{array}{ccccc}
\frac{2+v}{4} & \frac{2-v}{4} & 0 & \cdots & 0 \\
\frac{2-v}{4} & \frac{2+v}{4} & 0 & \cdots & 0
\end{array}\right)+ \\
& t\left(\begin{array}{cccccc}
1-\frac{v}{2} & \frac{v}{2} & 0 & 0 & \cdots & 0 \\
1-\frac{v}{2} & 0 & \frac{v}{2} & 0 & \cdots & 0
\end{array}\right)
\end{aligned}
$$

for $v \in[0,2]$. Therefore, $\Delta_{n}$ has no "holes". The case $n=2$ is handled in a similar way.

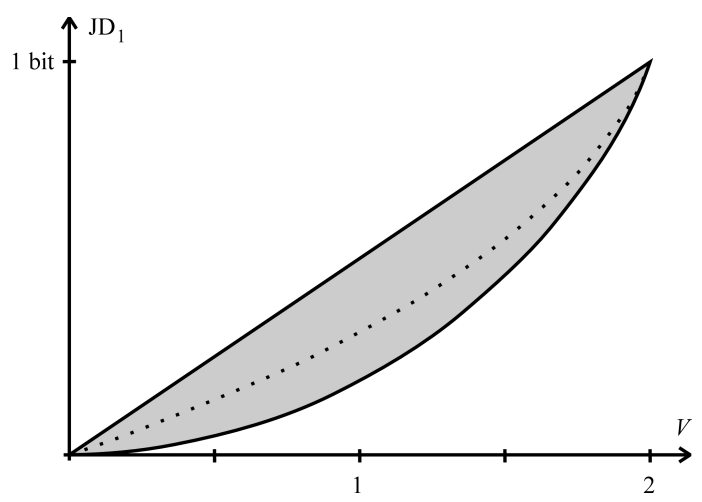

Figure 1: $V / \mathrm{JD}_{\alpha}$-diagram for $\alpha=1$ and $n \geq 3$ (the shaded region), and for $n=2$ (the region obtained by replacing the upper bounding curve by the dotted curve).

In Figure 1 we have depicted the $V / \mathrm{JD}_{\alpha}$-diagram for $\alpha=1$.

The bounds (18) and (19) give us the following proposition regarding the topology induced by $\left(\mathrm{JD}_{\alpha}\right)^{\frac{1}{2}}$. In the limiting case $\alpha \rightarrow 1$, this was proved in [5] by a different method.

Proposition 11. The space $\left(M_{+}^{1}(\mathbb{N}), \mathrm{JD}_{\alpha}^{1 / 2}\right)$ is a complete, bounded metric space for $\alpha \in(0,2]$, and the induced topology is that of convergence in total variation.

Proof: By expansion of $L(P, Q)$ given by 18 , in terms of the total variation $v$, one obtains the inequality

$$
\mathrm{JD}_{\alpha}(P, Q) \geq \frac{1}{\alpha-1} \sum_{j=1}^{\infty}\left(\begin{array}{c}
\alpha \\
2 j
\end{array}\right)\left(\frac{v}{2}\right)^{2 j} .
$$

Taking only the first term and bounding 19 , we get

$$
\begin{aligned}
\frac{1}{8} V^{2}(P, Q) & \leq \frac{\alpha}{8} V^{2}(P, Q) \\
& \leq \operatorname{JD}_{\alpha}(P, Q) \\
& \leq \frac{1}{\alpha-1}\left(\frac{1}{2}-\frac{1}{2^{\alpha}}\right)\|P-Q\|_{\alpha}^{\alpha} \\
& \leq \frac{\ln 2}{2} V(P, Q) .
\end{aligned}
$$

\section{B. Bounds on $\mathrm{QJD}_{\alpha}$}

With Theorem 9 we can bound QJD $_{\alpha}$ for $\alpha \in[1,2]$. We use the following two theorems.

Theorem 12 (49, Theorem 3.9). Let $\mathcal{H}$ be a Hilbert space, $\rho_{1}, \rho_{2} \in \mathcal{B}_{+}^{1}(\mathcal{H})$ and $\mathcal{M}:=\left\{M_{i} \mid i=1, \ldots, n\right\}$ be a measurement on $\mathcal{H}$. Then $S\left(\rho_{1} \| \rho_{2}\right) \geq D\left(P_{\mathcal{M}} \| Q_{\mathcal{M}}\right)$, where $P_{\mathcal{M}}, Q_{\mathcal{M}} \in M_{+}^{1}(n)$ and have point probabilities $P_{\mathcal{M}}(i)=\operatorname{Tr}\left(M_{i} \rho_{1}\right)$ and $Q_{\mathcal{M}}(i)=\operatorname{Tr}\left(M_{i} \rho_{2}\right)$, respectively.

Theorem 13 (38], Theorem 9.1). Let $\mathcal{H}$ be a Hilbert space,

$$
\rho_{1}, \rho_{2} \in \mathcal{B}_{+}^{1}(\mathcal{H})
$$

and $\mathcal{M}:=\left\{M_{i} \mid i=1, \ldots, n\right\}$ be a measurement on $\mathcal{H}$. Then $\left\|\rho_{1}-\rho_{2}\right\|_{1}=\max _{\mathcal{M}} V\left(P_{\mathcal{M}}, Q_{\mathcal{M}}\right)$, where $P_{\mathcal{M}}, Q_{\mathcal{M}} \in$ $M_{+}^{1}(n)$ and have point probabilities $P_{\mathcal{M}}(i)=\operatorname{Tr}\left(M_{i} \rho_{1}\right)$ and $Q_{\mathcal{M}}(i)=\operatorname{Tr}\left(M_{i} \rho_{2}\right)$, respectively.

Theorem 14. For $\alpha \in(0,2]$, for all states $\rho_{1}, \rho_{2} \in$ $\mathcal{B}_{+}^{1}(\mathcal{H})$, we have

$$
\begin{aligned}
s_{\alpha}\left(\frac{1}{2}\right)-s_{\alpha}\left(\frac{1}{2}+\frac{\left\|\rho_{1}-\rho_{2}\right\|_{1}}{2}\right) & \leq \operatorname{QJD}_{\alpha}\left(\rho_{1}, \rho_{2}\right) \\
& \leq \frac{\ln 2}{2}\left\|\rho_{1}-\rho_{2}\right\|_{1} .
\end{aligned}
$$

Proof: The lower bound is proved in the same way as [50, Theorem III.1], by making a reduction to the case of classical probability distributions by means of measurements. Let $\mathcal{M}$ be a measurement that maximizes $V\left(P_{\mathcal{M}}, Q_{\mathcal{M}}\right)$. Then from Theorem 13 we have $\left\|\rho_{1}-\rho_{2}\right\|_{1}=V\left(P_{\mathcal{M}}, Q_{\mathcal{M}}\right)$. Theorem 12 gives us

$$
\begin{aligned}
\operatorname{QJD}_{\alpha}\left(\rho_{1}, \rho_{2}\right) \geq & \frac{1}{2} D\left(P_{\mathcal{M}} \| \frac{P_{\mathcal{M}}+Q_{\mathcal{M}}}{2}\right) \\
& +\frac{1}{2} D\left(Q_{\mathcal{M}} \| \frac{P_{\mathcal{M}}+Q_{\mathcal{M}}}{2}\right) \\
= & \operatorname{JD}_{\alpha}\left(P_{\mathcal{M}}, Q_{\mathcal{M}}\right) .
\end{aligned}
$$

The result now follows from Theorem 9. The upper bound is proved the same way as we proved the classical bound. Introduce a 3 -dimensional Hilbert space $\mathcal{G}$ with basis vectors $|1\rangle,|2\rangle$ and $|3\rangle$. On $\mathcal{H} \otimes \mathcal{G}$ define the 
density matrices

$$
\begin{aligned}
\tilde{\rho}_{1}= & \frac{\rho_{1}+\rho_{2}-\left|\rho_{1}-\rho_{2}\right|}{2} \otimes|1\rangle\langle 1| \\
& +\frac{\rho_{1}-\rho_{2}+\left|\rho_{1}-\rho_{2}\right|}{2} \otimes|2\rangle\langle 2|, \\
\tilde{\rho}_{2}= & \frac{\rho_{2}+\rho_{1}-\left|\rho_{2}-\rho_{1}\right|}{2} \otimes|1\rangle\langle 1| \\
& +\frac{\rho_{2}-\rho_{1}+\left|\rho_{1}-\rho_{2}\right|}{2} \otimes|3\rangle\langle 3| .
\end{aligned}
$$

Let $\operatorname{Tr}_{\mathcal{G}}$ denote the partial trace $\mathcal{B}_{+}^{1}(\mathcal{H} \otimes \mathcal{G}) \rightarrow \mathcal{B}_{+}^{1}(\mathcal{H})$. Then $\operatorname{Tr}_{\mathcal{G}}\left(\tilde{\rho}_{1}\right)=\rho_{1}$ and $\operatorname{Tr}_{\mathcal{G}}\left(\tilde{\rho}_{2}\right)=\rho_{2}$. The matrices $\frac{\rho_{1}-\rho_{2}+\left|\rho_{1}-\rho_{2}\right|}{2}$ and $\frac{\rho_{2}-\rho_{1}+\left|\rho_{1}-\rho_{2}\right|}{2}$ are positive definite so

$$
\begin{aligned}
\left\|\tilde{\rho}_{1}-\tilde{\rho}_{2}\right\|_{1}= & \left.\operatorname{Tr}\left|\frac{\rho_{1}-\rho_{2}+\left|\rho_{1}-\rho_{2}\right|}{2} \otimes\right| 2\right\rangle\langle 2| \\
& -\frac{\rho_{2}-\rho_{1}+\left|\rho_{1}-\rho_{2}\right|}{2} \otimes|3\rangle\langle 3|| \\
= & \operatorname{Tr}\left(\frac{\rho_{1}-\rho_{2}+\left|\rho_{1}-\rho_{2}\right|}{2}\right) \\
& +\operatorname{Tr}\left(\frac{\rho_{2}-\rho_{1}+\left|\rho_{1}-\rho_{2}\right|}{2}\right) \\
= & \operatorname{Tr}\left|\rho_{1}-\rho_{2}\right|=\left\|\rho_{1}-\rho_{2}\right\|_{1} .
\end{aligned}
$$

According to the "quantum data processing inequality" [49. Theorem 3.10] we have

$$
\begin{aligned}
\mathrm{QJD}_{\alpha}\left(\rho_{1}, \rho_{2}\right) \leq \mathrm{QJD}_{1}\left(\tilde{\rho}_{1}, \tilde{\rho}_{2}\right) & \\
= & \frac{1}{2} \operatorname{Tr}\left(\frac{\rho_{1}-\rho_{2}+\left|\rho_{1}-\rho_{2}\right|}{2} \otimes|2\rangle\langle 2|\right) \ln 2 \\
+ & \operatorname{Tr}\left(\frac{\rho_{2}-\rho_{1}+\left|\rho_{1}-\rho_{2}\right|}{2} \otimes|3\rangle\langle 3|\right) \ln 2 \\
& =\frac{\ln 2}{2} \cdot\left\|\rho_{1}-\rho_{2}\right\|_{1} .
\end{aligned}
$$

\section{CONCLUSIONS AND OPEN PROBLEMS}

We studied generalizations of the (general) Jensen divergence and its quantum analogue. For $\alpha \in(1,2], \mathrm{JD}_{\alpha}$ was proved to be the square of a metric which can be embedded in a real Hilbert space. The same was shown to hold for $\mathrm{QJD}_{\alpha}$ restricted to qubit states or to pure states. Both these results were derived by evoking a theorem of Schoenberg's and showing that these quantities are negative definite.

Whether $\left(\mathrm{QJD}_{1}\right)^{\frac{1}{2}}$ is a metric for all mixed states remains unknown. However, based on a large amount of numerical evidence, we conjecture the function $A \rightarrow$ $\operatorname{Tr}\left(e^{A}\right)$ to be exponentially convex for density matrices $A$. Proving this would imply that $\mathrm{QJD}_{\alpha}$ is negative definite for $\alpha \in(0,2]$, and hence the square of a metric that can be embedded in a real Hilbert space.

\section{ACKNOWLEDGEMENTS}

We are greatly indebted to Flemming Topsøe. This work mainly extends his basic result jointly with Bent Fuglede presented at the conference ISIT 2004 [4], where you find the basic result on isometric embedding in Hilbert space related to $\mathrm{JD}_{1}$. Flemming has supplied us with many valuable comments and suggestions. In particular Section V is to a large extent inspired by unpublished results of Flemming.

Jop Briët is partially supported by a Vici grant from the Netherlands Organization for Scientific Research (NWO), and by the European Commission under the Integrated Project Qubit Applications (QAP) funded by the IST directorate as Contract Number 015848. Peter Harremoës has been supported by the Villum Kann Rasmussen Foundation, by Danish Natural Science Research Council, by INTAS (project 00-738) and by the European Pascal Network.
[1] D. M. Endres and J. E. Schindelin. A new metric for probability distributions. IEEE Trans. Inf. Theory, 49:1858-60, 2003.

[2] R. G. Gallager. Information Theory and Reliable Communication. Wiley and Sons, New York, 1968.

[3] J. Lin and S. K. M. Wong. A new directed divergence measure and its characterization. Int. J. General Systems, 17:73-81, 1990.

[4] J. Lin. Divergence Measures Based on the Shannon Entropy. IEEE Trans Inform. Theory, 37:145-151, 1991.

[5] F. Topsøe. Some inequalities for information divergence and related measures of discrimination. IEEE Trans. Inform. Theory, 46:1602-1609, 2000.

[6] R. Sibson. Information radius. Z. Wahrs und verw Geb., 14:149-160, 1969.

[7] A. K. C. Wong and M. You. Entropy and distance of random graphs with application to structural patternrecognition. IEEE Trans. Pattern Anal. Machine Intell., 7:599-609, 1985.

[8] O. A. Rosso, H. A. Larrondo, M. T. Martin, A. Plastino, and M. A. Fuentes. Distinguishing noise from chaos. Phys. Rev. Lett., 99(15):154102, 2007.

[9] R. El-Yaniv, S. Fine, and N. Tishby. Agnostic classification of Markovian sequences. NIPS, MIT-Press, pages 465-471, 1997.

[10] J. Burbea and C. R. Rao. On the convexity of some divergence measures based on entropy functions. IEEE Trans. Inform. Theory, 28:489-495, 1982.

[11] C. Tsallis. Possible generalization of Boltzmann-Gibbs statistics. J. Stat. Physics, 52:479, 1988.

[12] J. Lindhard and V. Nielsen Studies in Dynamical Systems Kongelige Danske Videnskabernes Selskab, 
Matematisk-Fysiske Meddelser, 38(9):1-42, 1971.

[13] M. Figueiredo A. Martins, P. Aguiarz. Tsallis kernels on measures. IEEE Information Theory Workshop, pages 298-302, 2008.

[14] A. Martins, P. Aguiar, and M. Figueiredo. Nonextensive Generalizations of the Jensen-Shannon Divergence. Submitted, Apr 2008.

[15] A. F. T. Martins, M. A. T. Figueiredo, P. M. Q. Aguiar, N. A. Smith, and E. P. Xing. Nonextensive entropic kernels. In ICML '08: Proceedings of the 25th international conference on Machine learning, pages 640-647, New York, NY, USA, 2008. ACM.

[16] P. M. Q. Aguiar N. A. Smith E. P. Xing A. F. T. Martins, M. A. T. Figueiredo. Nonextensive entropic kernels. Tech. report CMU-ML-08-106, Carnegie Mellon University, 2008.

[17] W. K. Wootters. Statistical distance and Hilbert space. Phys. Rev. D, 23(2):357-362, Jan 1981.

[18] S. L. Braunstein and C. M. Caves. Statistical distance and the geometry of quantum states. Phys. Rev. Lett., 72(22):3439-3443, May 1994.

[19] J. Lee, M. S. Kim, and Č. Brukner. Operationally invariant measure of the distance between quantum states by complementary measurements. Phys. Rev. Lett., 91(8):087902, Aug 2003.

[20] A. P. Majtey, P. W. Lamberti, M. T. Martin, and A. Plastino. Wootters' distance revisited: a new distinguishability criterium. Eur. Phys. J. D, 32:413-419, 2005.

[21] P. W. Lamberti, A. P. Majtey, A. Borras, M. Casas, and A. Plastino. On the metric character of the quantum Jensen-Shannon divergence. Phys. Rev. A, 77(5):052311, 2008.

[22] B. Schumacher and M. D. Westmoreland. Relative entropy in quantum information theory. In S. Lomonaco, editor, Quantum Computation and Quantum Information: A Millenium Volume. American Mathematical Society Contemporary Mathematics series, 2001.

[23] A. P. Majtey, P. W. Lamberti, and D. P. Prato. JensenShannon divergence as a measure of distinguishability between mixedquantum states. Phys. Rev. A, 72(5):052310, 2005.

[24] P. W. Lamberti, M. Portesi, and J. Sparacino. A natural metric for quantum information theory. arXiv:quantph/080\%.0583v1, Jul 2008.

[25] T. Cover and J. A. Thomas. Elements of Information Theory. Wiley, 1991.

[26] A. Rényi. On Measures of Entropy and Information. In Proc. 4th Berkeley Symp. Math. Statist. and Prob. 1:547561, Univ. Calif. Press, Berkely, 1961.

[27] J. H. Havrda and F. Charvat. Quantification methods of classification processes: concepts of structural $\alpha$ entropy. Kybernatica, 3:30-35, 1967.

[28] J. Aczél and Z. Daróczy On Measures of Information and their Characterization. Mathematics in Science and Engineering vol. 115, Academic Press, New York, 1975.

[29] A. S. Holevo. Information theoretical aspects of quantum measurements. Probl. Inf. Transm., 9:110-118, 1973.

[30] A. S. Holevo. The capacity of quantum channel with general signal states. IEEE Trans. Inform. Theory, 44:269273, 1998.

[31] B. Schumacher and M. D. Westmoreland. Sending classi- cal information via noisy quantum channels. Phys. Rev. A, 56(1):131-138, Jul 1997.

[32] B. Fuglede and F. Topsøe. Jensen-Shannon divergence and Hilbert space embedding. In Proceedings 2004 International Symposium on Information Theory, page 31, 2004.

[33] F. Topsøe. An information theoretical identity and a problem involving capacity. Studia Scientiarum Mathematicarum Hungarica, 2:291-292, 1967.

[34] F. Topsøe. Basic concepts, identities and inequalities the toolkit of information theory. Entropy, 3(3):162-190, 2001.

[35] B. Schumacher. Quantum coding. Phys. Rev. A, 51:27382747, April 1995.

[36] B. Schumacher and M. D. Westmoreland. Indeterminatelength quantum coding. Phys. Rev. A, 64(4):042304, Sep 2001.

[37] M. J. Donald. Further results on the relative entropy. Math. Proc. Cam. Phil. Soc., 101:363, 1987.

[38] M. Nielsen and I. L. Chuang. Quantum Computation and Quantum Information. Cambridge University Press, Cambridge, 2000.

[39] M. Ohya, D. Petz. Quantum entropy and its use. Texts and Monographs in Physics. Springer-Verlag, Berlin, 1993.

[40] M. Laurent M. Deza. Geometry of Cuts and Metrics. Springer-Verlag, Berlin, 1997.

[41] L. M. Blumenthal. Theory and Applications of Distance Geometry. Oxford University Press, London, 1953.

[42] K. Menger. Géométrie Générale. Gauthier-Villars, Paris, 1954.

[43] I. J. Schoenberg. Remarks to Maurice Fréchet's article "Sur la définition axiomatique d'une classe d'espace distanciés vectoriellement applicable sur l'espace de Hilbert". Annals of Mathematics, 36:724-732, 1935.

[44] I. J. Schoenberg. Metric spaces and positive definite functions. Trans. Amer. Math. Soc., 44:522-536, 1938.

[45] A. E. Nussbaum. Radial exponentially convex functions. Journal d'Analyse Mathématique, 25(1):277-288, December 1972 .

[46] B. Fuglede. Spirals in Hilbert space: with an application in information theory. Expo. Math., 23:23-45, 2005.

[47] B. Fuglede and F. Topsøe. Jensen-Shannon divergence and Hilbert space embedding. In Proceedings 2004 International Symposium on Information Theory, page 31, 2004.

[48] C. Berg, J. P. R. Christensen, and P. Ressel. Harmonic Analysis on Semigroups. Springer-Verlag, New York, 1984.

[49] D. Petz. Quantum information theory and quantum statistics. Springer, Berlin, 2008.

[50] H. Klauck, A. Nayak, A. Ta-Shma, and D. Zuckerman. Interaction in quantum communication. IEEE Transactions on Information Theory, 53(6):1970-1982, 2007.

[51] This deviates from the notation used in [21. We do this for the sake of consistency with regard to the notation for probability distributions.

[52] The calculation of the Taylor expansion involves a lot of computations but are easily performed using Maple or similar symbol manipulation program. 